\title{
Some Aspects of Astronomy at Maidanak Observatory
}

\author{
Alisher S. Hojaev \\ Astronomical Institute, Astronomicheskaya 33, Tashkent, Uzbekistan
}

\begin{abstract}
A brief overview for some aspects of astronomical investigations at Maidanak is given. An excursus is made into its history and past research. Future plans and their requirements are described.
\end{abstract}

\section{Introduction}

By now it has been generally recognized that Central Asia, especially its southwestern part, may be one of the most promising regions for ground-based astronomy. The necessity to choose a place for the $6.0 \mathrm{~m}$ telescope (BTA) have stimulated astroclimatic (astronomical site testing) campaigns in the region of the Tian-Shan, Pamir and Alay mountains in the early 1960s. It was found that the relatively dry air and the availability of suitable sites at altitudes of about $3 \mathrm{~km}$ above sea level provide high transparency in the optical, as well as in the far-IR and submillimeter range, with a maximum number of clear nights (at least in the area of the FSU), maximum sky stability, and a low amount of precipitation.

\section{The Observatory}

An analysis of the geographic, climatic, and atmospheric conditions for about a few dozen different sites in this region revealed Mt. Maidanak ( $\mathrm{E} 66^{\circ} 56^{\prime}$; N $38^{\circ} 41^{\prime}$ ) to be the best for an astronomical observatory. This isolated summit is located on one of the parallel ridges belonging to the Pamir and Alay mountain region in the south-eastern part of Uzbekistan with an altitude of about $2600 \mathrm{~m}$. Fig. 1 presents the location of Maidanak on a world map. The first astroclimatic measurements started in August 1969 and became regular in April 1970 (Hetselius 1972). The long-term explorations have clearly shown that Maidanak has a seeing in the sub-arcsecond range (Shevchenko 1973, Shcheglov \& Gur'yanov 1991, Gur'yanov et al. 1992), a large number of clear nights (Novikova 1970, Gladyshev \& Shirokova 1987), a low night sky background (Kardopolov \& Filip'ev 1979), and a high optical transparency (Zheleznyakova 1984), thus making it one of the most preferable sites worldwide for ground-based astronomy. Final tests by the ESO Differential Image Motion Monitor (Sarazin \& Roddier 1990), which was used for site testing in Chile, and a Generalized Seeing Monitor (Martin et al. 1994) confirmed these estimations (e.g. Sarazin 1999, Egamberdiev et al. 2000). 


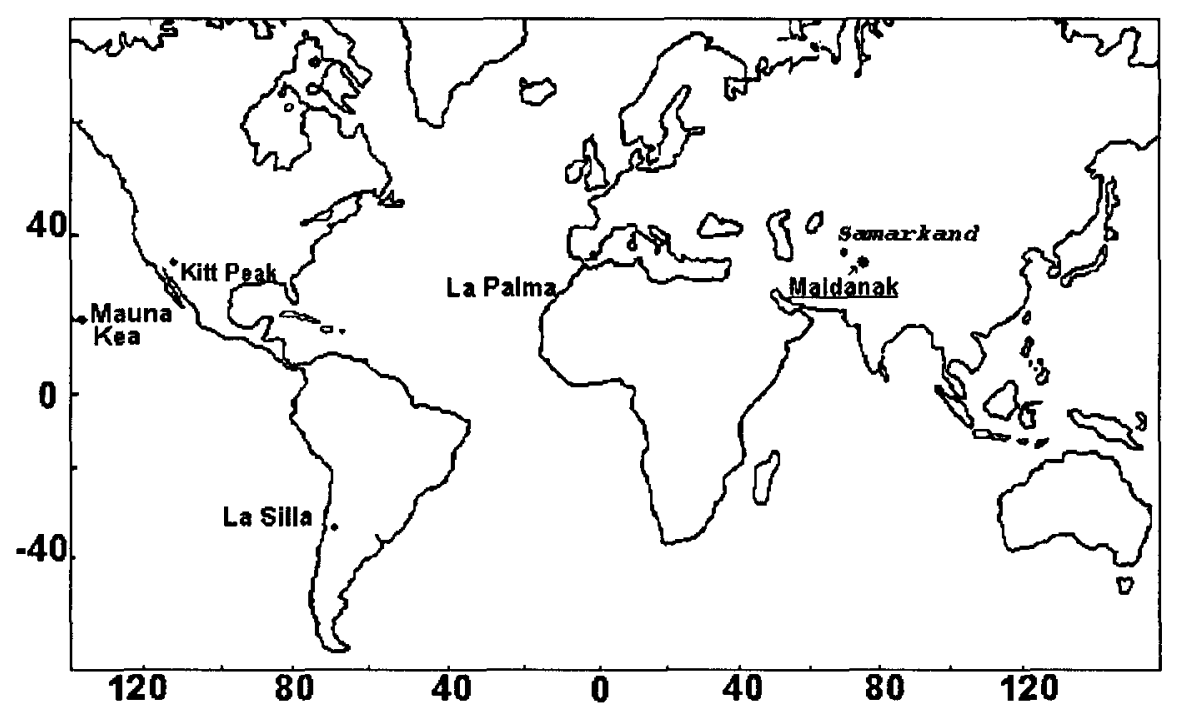

Figure 1. Location of the Maidanak observatory

In fact there are two summits - Eastern and Western Maidanak, about $5 \mathrm{~km}$ apart with the eastern peak being a bit higher. After ensuring the high quality of atmospheric conditions, a satellite laser ranging station was established on the Eastern summit (Scott 1995). Researchers from the Astronomical Institutes of Uzbekistan, Ukraine, and Lithuania carried out observations with a few small telescopes (two $60 \mathrm{~cm}$, one $48 \mathrm{~cm}$, two $40 \mathrm{~cm}$ and some smaller ones) installed on the same summit. The Western summit hosted the observational facilities of the Sternberg Astronomical Institute of the Moscow State University, Astronomical observatories of Lithuania, Ukraine, St.Petersburg University and others. All these astronomical facilities including their infrastructure are now under the jurisdiction of the Astronomical Institute of Uzbekistan and are operated according to agreements between the astronomical institutions of Uzbekistan, Russia, Ukraine and Lithuania. Steps are being made to arrange an international observatory at Maidanak. Presently the observatory facilities consist of a $1.5 \mathrm{~m}$ and a $1.0 \mathrm{~m}$ telescope as well as 9 other small telescopes with apertures of $60 \mathrm{~cm}$ or less. A general view of the observatory on the Western summit is given in Fig. 2.

\section{Research : A Brief Overview}

The first astronomical investigations at Eastern Maidanak started in the early 1970s. In 1971 a small Schmidt telescope was installed on the summit and since 1972 this telescope has been used for observations of T-associations (Isakov 1975). In the same year, a 16 " Cassegrain reflector for photometry received its first light there (Shevchenko 1974). Later on, two $60 \mathrm{~cm}$ Zeiss600 telescopes, a 


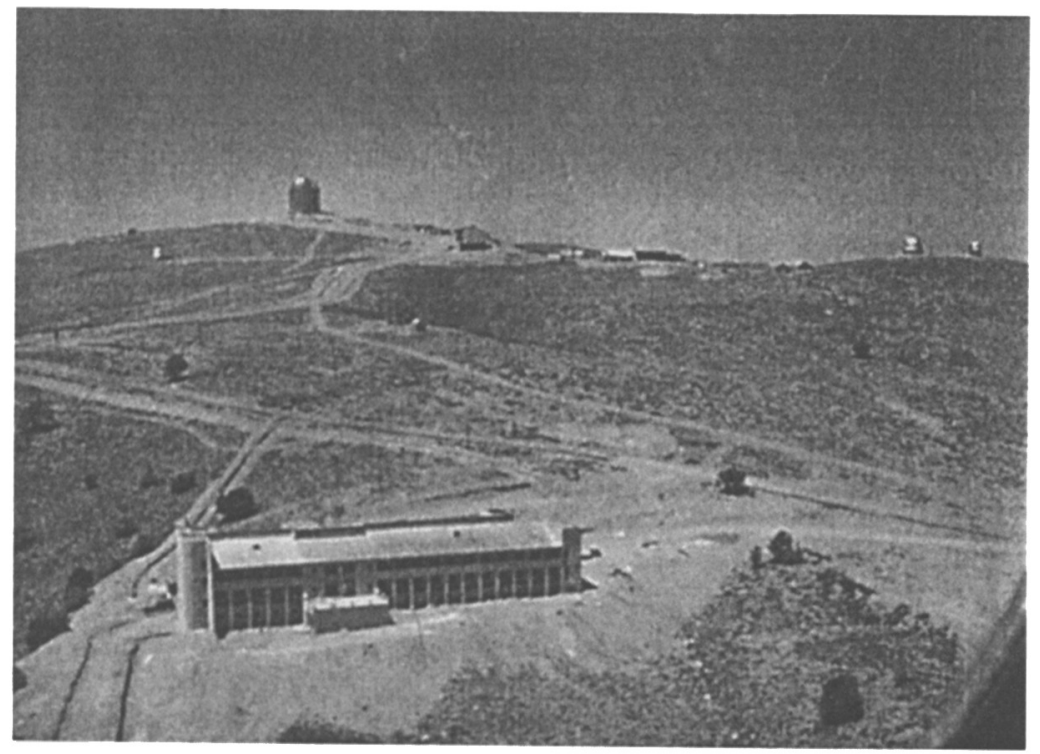

Figure 2. A bird's-eye view of Western Maidanak

$48 \mathrm{~cm}$ LOMO AZT-14, a $40 \mathrm{~cm}$ and different other smaller telescopes started their operation.

Some of these telescopes, equipped with photomultiplier tube photon counting photometers, which have been adapted to the international UBVRI system, are used for monitoring of variable stars, such as T Tauri stars, Herbig AeBe stars and other PMS-stars, Cepheids, eclipsing binaries, patrolling of stellar flares and surveying of other unique objects. A large photometric data bank has been collected since the mid 1970s. Ukrainian astronomers also carried out photometric and polarimetric observations of solar system planets (especially Jupiter and Saturn), their satellites and Saturn's rings using one of the Zeiss600 telescopes. Baltic colleagues in cooperation with visiting scientists carried out stellar photometry of star-forming regions as well as other regions in the Vilnius and VilGen systems by the $40 \mathrm{~cm}$ telescope. In the late $1970 \mathrm{~s}$ and in the early 1980 s, researchers from the Astronomical Institutes of Uzbekistan and Byurakan Astrophysical observatory of Armenia have used the local network of observatory telescopes (usually 3 ) for simultaneous multiband patrol of flare stars (UV Cet, EV Lac, Pleiades campaigns). Such local telescope networks were also used to observe X-ray sources (Cyg X-1, Cyg X-2, Her X-1 etc).

During that period, successful observations in the infrared range were also made: near-IR spectroscopy of PMS-stars, mainly HAeBe stars and T Tauri stars, using an image intensifier with an oxygen-silver-cesium photocatode (e.g. Kotyshev 1988) and JHK photometric measurements of IR-sources, based on lead-sulfide cells, both cooled by dry ice. Moreover, spectroscopy of HAeBe stars in both optical and UV wavelengths was attempted using the image intensifier with a multi-alkaline photocatode. 


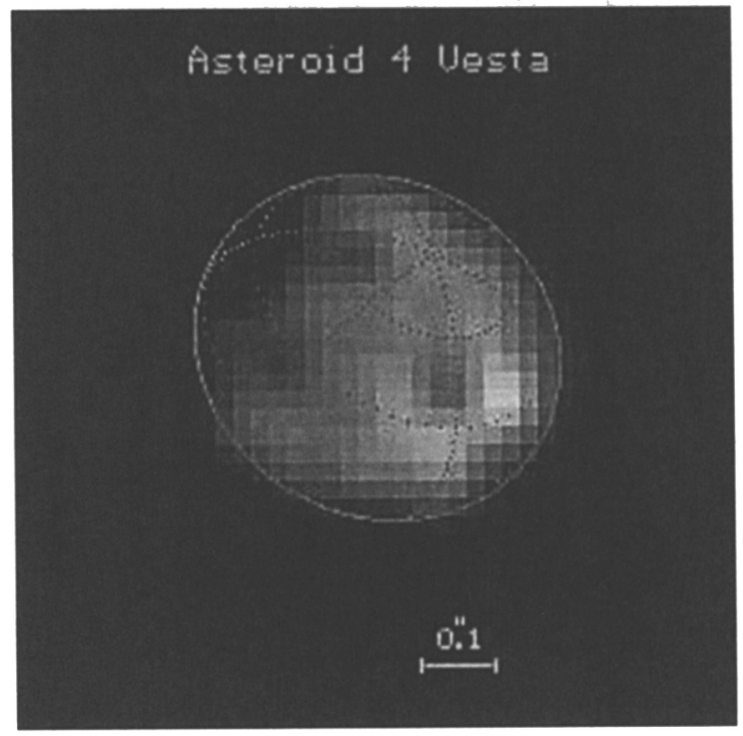

Figure 3. A speckle frame synthesized image (with $R=0.11^{\prime \prime}$ ) of the asteroid 4 Vesta; for details, see Tsvetkova et al. (1991).

The installation of the $1.0 \mathrm{~m}$ Zeiss 1000 telescope on the Western summit has opened new possibilities, particularly in high-resolution imaging of celestial bodies. As an example an image of the asteroid 4 Vesta (Tsvetkova et al. 1991) is presented in Fig. 3, which reveals the excellent seeing conditions of the site. This telescope had also an equipment for Vilnius system photometry, used by the Baltic astronomers for surveying the Galaxy stellar content.

Meanwhile a $1.5 \mathrm{~m}$ AZT-22 telescope, constructed by LOMO and installed at Western Maidanak, obtained better images due to the high optical quality of its main mirror (near diffraction limit). In July 1994 most of our telescopes were used to observe the crash of comet Shoemaker-Levy 9/P on Jupiter. Polarimetric measurements carried out by the $1.5 \mathrm{~m}$ and $1.0 \mathrm{~m}$ telescopes in addition to multicolor imaging, revealed significant differences in the polarization of the impact sites (Dudinov et al. 1995, Fig. 4). Based on these observations a consistent physical model of the phenomena was developed (Shevchenko et al. 1996). In addition, using the $1.0 \mathrm{~m}$ Zeiss telescope with the special image intensifying camera we observed young stellar objects, such as V1331 Cyg (Hojaev \& Zheleznyak 1998). The star and its circumstellar environment are shown in Fig. 5. An outflow phenomenon in this relatively massive star detected in the optical range (Hojaev 1999) was corroborated by other authors. An absolute elements and physical models were defined for close binaries, e.g. IT Cas (Lacy et al. 1997), V364 Lac (Torres et al 1999), WZ Cep (Djuracevic et al. 1997), see also Zakirov et al. (1996). 

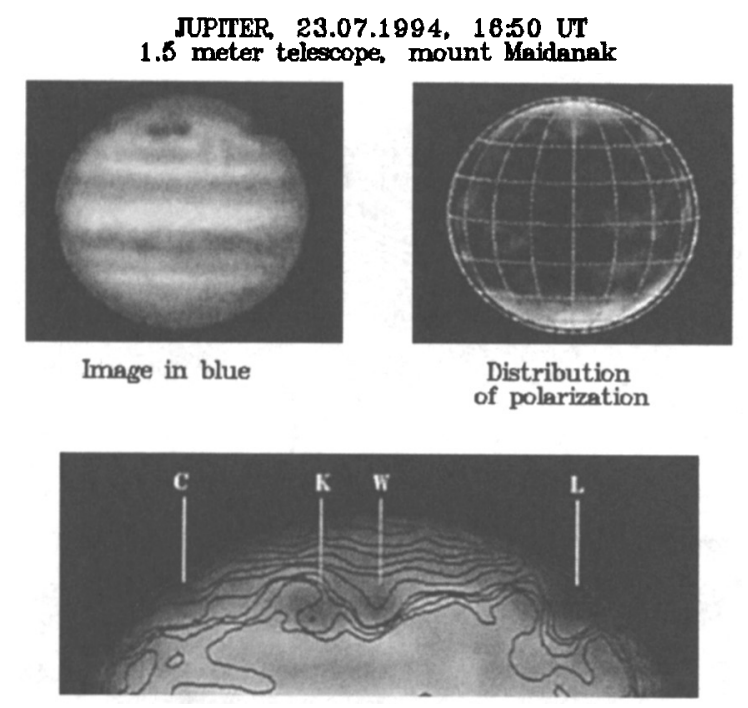

Polarization of South Polar Region in more detailes (contours). The range of polarization variations is from $2 \%$ to $12 \%$ Image in blue is presented in half-tones

Figure 4. Jupiter during the CSL9/P impact

\section{Future Exploration}

Its specific geographical position covering the gap between observatories in Chile, Hawaii and the Canary islands (see Fig. 1), makes Maidanak very favorable for projects requiring uninterrupted observations of celestial bodies. The excellent seeing conditions render images with high angular resolution.

New developments demand that we update our telescopes by new backilluminated chips, digital photometers, effective high-resolution multidimensional spectrographs and other new techniques in focal plane instrumentation. It is essential to establish a broad-band internet connection using a satellite link at the observatory to obtain necessary information in time, to receive and send data on-line, to join international networks of telescopes, to arrange for appropriate ground based support for space born observations etc.

Maidanak is worthy of installing a new generation of telescopes with larger apertures as well as automatic and robotic telescopes, demanding high capability internet links for remote observations. Existing telescopes can be transfered to automatic or at least semiautomatic ones. The site also provides the conditions for optical interferometry, and steps are being made to gradually improve the instrumentation and infrastructure. Lately a few CCD cameras including LNcooled ones have started their operations mainly for monitoring GLS, AGNs and QSOs within the framework of international cooperation. Our aim is to make such collaboration more intensive and extensive as well. 


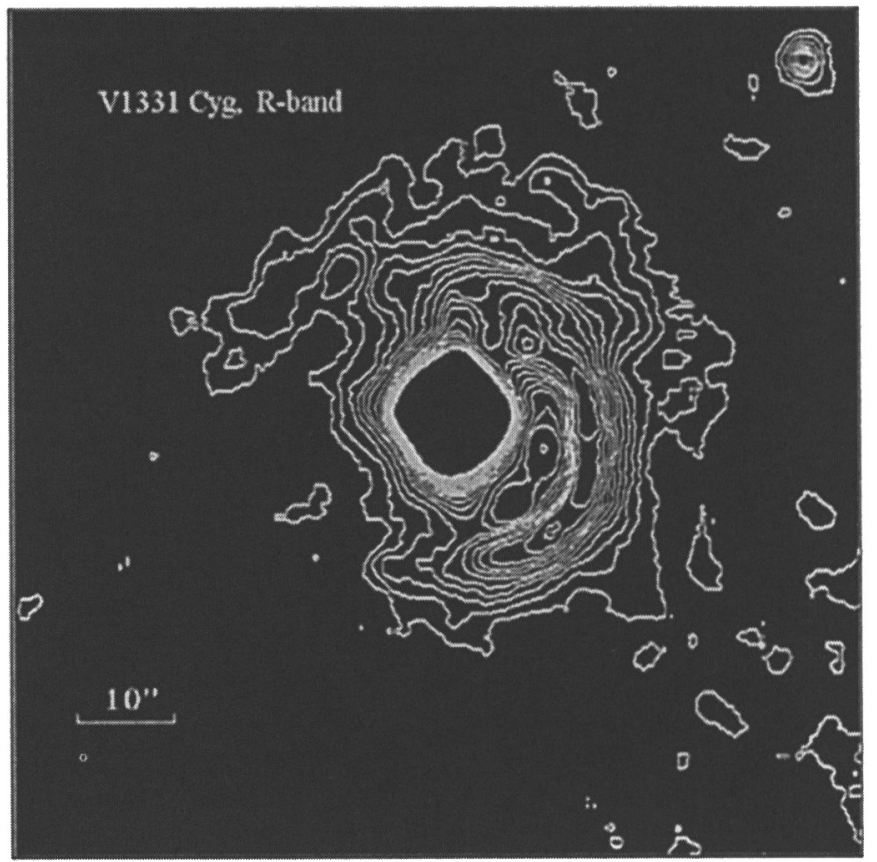

Figure 5. The PMS-star V1331 Cyg and its environment

\section{Conclusion}

We have described the outstanding position of Maidanak for ground-based astronomy and have presented some previous and ongoing research as well as our future plans for improving the research capabilities at this site. It is not possible here to give a complete and detailed description, but we hope that we provided some idea about this observatory which deserves a place among the best ones. A close collaboration with astronomers and astronomical institutions world-wide is desired and their possible support by, for instance, the Maidanak Foundation, will be mutually beneficial.

Acknowledgments. The author is thankful to Prof. W.P. Chen for his generous support, discussions and interest in the paper. We are also grateful to Mr. Denis Ustimenko for his kind software assistance in preparing the manuscript.

\section{References}

Djurasevic, G. et al. 1998, A\&AS, v.131, 17

Dudinov, V.N. et al. 1995, in ESO Conf. \& Workshop Proceed., No52, 323

Egamberdiev, S.A. et al. 2000, ESO Preprint No1381

Gladyshev, S.A.\& Shirokova, M.G. 1987 in Methods for Increasing the Efficiency of Optical Telescopes (Moscow: MSU), 45 
Gur'yanov, A.E. et al. 1992, A\&A, 262, 373

Hetselius, V.G. 1972, in Young Stellar Complexes and Astroclimate (Tashkent: Fan), 137

Hojaev, A.S. 1999, New Astronomy Reviews, 43, 431

Hojaev, A.S. \& Zheleznyak, A.P. 1997 in Low Mass Star Formation from Infall to Outflow (Grenoble: IAU), 218

Isakov, I.S. 1975, in Study of Extremely Young Stellar Complexes (Tashkent: Fan), 143

Kardopolov, V.I. \& Filip'ev, G.K. 1979, Pisma v AZh, 5, 106

Kotyshev, V.V. 1988, Tsirk. AI, N127(474), 18

Lacy, C.H.S. et al. 1997, AJ, 114, 1206

Martin, F. et al. 1994, A\&AS, 108, 173

Novikova, G.V. 1970, in Atmospheric Optics (Moscow: Nauka), 10

Sarazin, M. \& Roddier, F. 1990, A\&A, 227, 294

Sarazin, M. 1999, http://www.eso.org/gen-fac/pubs/astclim/espas/maidanak/

Scott, W.B. 1995, Av.Week Space Tech., 142, N20 (15 May), 68

Shcheglov, P.V. \& Gur'yanov, A.E. 1991, Soviet Ast., 68, 632

Shevchenko, V.S. 1973, AZh, 50, N3, 632

Shevchenko, V.S. 1974, in 100 years for Astronomical institute of Uzbek Academy of Sciences (Tashkent: Fan), 113

Shevchenko, V.V. et al. 1996, Astron.Vestnik (Solar System Research), 30, No 2,101

Torres, G. et al. 1999, AJ, 118, 1831

Tsvetkova, V.S. et al. 1991, Icarus, 92, 342

Zakirov, M.M. et al. 1996, Journal Korean Astron. Soc., 29, S245

Zheleznyakova, A.I. 1984, in Astroclimate and Efficiency of Telescopes (Moscow: Nauka), 55 